\title{
An Accurate Quartic Force Field, Fundamental Frequencies, and Binding Energy for the High Energy Density Material $T_{d} \mathrm{~N}_{4}$
}

\author{
Timothy J. Lee ${ }^{1}$ \\ MST27b-1, NASA Ames Research Center, Moffett Field, CA 94035-1000
}

\author{
Jan M. L. Martin ${ }^{2}$ \\ Department of Organic Chemistry, Kimmelman Building, Room 262, \\ Weizmann Institute of Science, 76100 Rehovot, Israel
}

\begin{abstract}
The CCSD(T) method has been used to compute a highly accurate quartic force field and fundamental frequencies for all ${ }^{14} \mathrm{~N}$ and ${ }^{15} \mathrm{~N}$ isotopomers of the high energy density material $T_{d} \mathrm{~N}_{4}$. The computed fundamental frequencies show beyond doubt that the bands observed in a matrix isolation experiment by Radziszewski and coworkers are not due to different isotopomers of $T_{d} \mathrm{~N}_{4}$. The most sophisticated thermochemical calculations to date yield a $\mathrm{N}_{4} \rightarrow 2 \mathrm{~N}_{2}$ heat of reaction of $182.22 \pm 0.5 \mathrm{kcal} / \mathrm{mol}$ at $0 \mathrm{~K}$ $(180.64 \pm 0.5$ at $298 \mathrm{~K})$. It is hoped that the data reported herein will aid in the ultimate detection of $T_{d} \mathrm{~N}_{4}$.
\end{abstract}

\section{Introduction}

Beginning in the early $1990 \mathrm{~s}$, theoretical quantum mechanical calculations identified tetrahedral $\left(T_{d}\right) \mathrm{N}_{4}$ as a potentially useful novel rocket fuel for a number of reasons [1-11]. First, a considerable amount of energy is released upon dissociation into two $\mathrm{N}_{2}$ molecules (183 kcal/mol [1]; see also Ref. 2 in [12]). Second, there is a large barrier to dissociation into two $\mathrm{N}_{2}$ molecules, and the curve crossing to the lowest triplet state was $13 \mathrm{kcal} / \mathrm{mol}$ above the $T_{d} \mathrm{~N}_{4}$ minimum making it possible to handle $T_{d} \mathrm{~N}_{4}$. Third, the main dissociation product, $\mathrm{N}_{2}$, is environmentally benign. Further studies examined the spin-orbit crossing probability as well as the barrier to dissociation, and concluded that indeed $T_{d} \mathrm{~N}_{4}$ held promise as a novel rocket fuel should it be possible to synthesize the compound.

Recently, there has been renewed interest in the high energy density material $T_{d}$ $\mathrm{N}_{4}$ [12-15]. Aside from the study by Radziszewski and coworkers, which will be discussed below, the excited electronic states of $T_{d} \mathrm{~N}_{4}$ were investigated [12,14]. In one of these studies a novel approach to the synthesis of $T_{d} \mathrm{~N}_{4}$ was proposed [14], which is based on combining two $\mathrm{N}_{2}$ molecules in bound quintet excited electronic states. While this new approach appears promising and is the first proposed method which targets the electronic structure of the reactants to be similar to the desired end result, $T_{d} \mathrm{~N}_{4}$, it has yet to be attempted experimentally. In addition, Cacace $e t$ al. [15] have very recently reported the synthesis of a tetranitrogen compound, although this is not $T_{d} \mathrm{~N}_{4}$. Further, this compound is not a high energy density material, and since it has a chain-like structure with two short N-N bonds, it is difficult to see how it will be useful in making $T_{d} \mathrm{~N}_{4}$.

\footnotetext{
' tjlee@mail.arc.nasa.gov

2 comartin@wicc.weizmann.ac.il
} 
In the summer of 2000 it appeared that at last some progress had been made when a study was published by Radziszewski and coworkers in which they tentatively claimed to have made $T_{d} \mathrm{~N}_{4}$ [13]. The claim was based on observation of the one infrared active band of $T_{d} \mathrm{~N}_{4}$ and its full ${ }^{15} \mathrm{~N}$ isotopomer. However, the isotopic shift of the band did not agree with theoretical calculations with the experimental value being $36.7 \mathrm{~cm}^{-1}$ while theory placed this shift at $31.6 \mathrm{~cm}^{-1}[13,14]$. Radziszewski and coworkers were also concerned about their inability to observe IR bands from any of the mixed isotopomers. However, as we have recently explained [14], this is not surprising since observation of the ${ }^{14} \mathrm{~N}_{4}$ and ${ }^{15} \mathrm{~N}_{4}$ isotopomers was at their instrumentation limits, and the IR bands for the mixed isotopomers would have significantly reduced intensities due to a splitting of the triply degenerate vibration. Thus the main concern from the work in Ref. [13] is the disgreement between theory and experiment for the ${ }^{14} \mathrm{~N}_{4}$ to ${ }^{15} \mathrm{~N}_{4}$ isotopic shift. Since matrix isolation was used in Ref. [13], it is possible to have bands shift due to interaction with the matrix, but it is highly unlikely that any shift would be different for ${ }^{14} \mathrm{~N}$ and ${ }^{15} \mathrm{~N}$ isotopomers, so this possible source of error for the isotopic shift can be eliminated. Another source of error, which is more of a possibility, is that the isotopic shift determined from theory is in error since only harmonic frequencies were used. We have addressed this issue in the present study by computing a highly accurate quartic force field for $T_{d} \mathrm{~N}_{4}$ using the singles and doubles coupled-cluster method that includes a perturbational correction for triple excitations, CCSD(T) [16]. This method has been shown to yield highly accurate vibrational transition frequencies [17-20], and it should allow us to determine definitively whether the bands observed by Radziszewski and coworkers are indeed due to $T_{d} \mathrm{~N}_{4}$.

The theoretical approach is given in the next section while the results and discussion are presented in the following section. Conclusions are given in the final section.

\section{Theoretical Approach}

The CCSD(T) electronic structure method was used to obtain a quartic force field for $T_{d} \mathrm{~N}_{4}$. In these calculations, Dunning's [21] correlation consistent basis set that includes up through $g$-functions on nitrogen, cc-pVQZ, was used. In order to account for the effects of core-correlation, a $\operatorname{CCSD}(\mathrm{T})$ quadratic force field was obtained with Woon and Dunning's [22] cc-pCVQZ basis set. In all CCSD(T) calculations, only spherical harmonic components of $d, f$, and $g$-functions were used. As noted previously, the $\operatorname{CCSD}(\mathrm{T})$ method has been shown to yield highly accurate force fields and vibrational frequencies for a range of molecular systems [17-20]. All CCSD(T) electronic structure calculations have been performed with the MOLPRO program package [23].

The equilibrium structure of the molecule was determined such that the residual internal coordinate gradient was less than $1 \times 10^{-6}$ atomic units. The quartic force field was then determined in symmetry internal coordinates. These coordinates are given by,

$$
\begin{aligned}
& \mathrm{S}_{1}\left(\mathrm{a}_{1}\right)=1 / \sqrt{6}\left(\mathrm{r}_{1}+\mathrm{r}_{2}+\mathrm{r}_{3}+\mathrm{r}_{4}+\mathrm{r}_{5}+\mathrm{r}_{6}\right) \\
& \mathrm{S}_{2 \mathrm{a}}(\mathrm{e})=1 / \sqrt{ } 12\left(2 \mathrm{r}_{1}-\mathrm{r}_{2}-\mathrm{r}_{3}+2 \mathrm{r}_{4}-\mathrm{r}_{5}-\mathrm{r}_{6}\right) \\
& \mathrm{S}_{2 \mathrm{~b}}(\mathrm{e})=1 / \sqrt{ } 4\left(\mathrm{r}_{2}-\mathrm{r}_{3}+\mathrm{r}_{5}-\mathrm{r}_{6}\right)
\end{aligned}
$$


$\mathrm{S}_{3 x}\left(\mathrm{t}_{2}\right)=1 / \sqrt{ } 2\left(\mathrm{r}_{2}-\mathrm{r}_{5}\right)$,

$S_{3 y}\left(t_{2}\right)=1 / \sqrt{2}\left(r_{3}-r_{6}\right)$,

$\mathrm{S}_{3 z}\left(\mathrm{t}_{2}\right)=1 / \sqrt{2}\left(\mathrm{r}_{1}-\mathrm{r}_{3}\right)$,

The numbering of bonds is such that if $r_{1}$ connects one pair of atoms then $r_{4}$ connects the other pair and similarly for the $\left\{r_{2}, r_{5}\right\}$ and $\left\{r_{3}, r_{6}\right\}$ pairs. These are the same symmetry coordinates used previously for a quartic force field calculation for the tetrahedral molecule $\mathrm{Be}_{4}$ [24], except that a typographical error for the $\mathrm{S}_{2 \mathrm{a}}$ coordinate has been corrected here. The quartic force field constants, given in Table 1, correspond to an unconstrained summation also as used previously in Ref. [24]. Only the symmetry unique force constants are listed in Table 1 --- see Ref. [24] for symmetry relationships for the quadratic, cubic, and quartic force constants. Also presented in Table 1 are the quadratic force constants obtained at the $\operatorname{CCSD}(\mathrm{T}) / \mathrm{cc}-\mathrm{pCVQZ}$ level of theory. The anharmonic spectroscopic analysis was performed with a modified version of the SPECTRO [25] program.

\section{Results and Discussion}

\section{A. Anharmonic Constants and Accurate Vibrational Transition Energies}

The CCSD(T)/cc-pVQZ quartic force field given in Table 1 was used to obtain fundamental vibrational frequencies for the ${ }^{14} \mathrm{~N}_{4},{ }^{15} \mathrm{~N}_{4},{ }^{14} \mathrm{~N}_{3}{ }^{15} \mathrm{~N},{ }^{14} \mathrm{~N}^{15} \mathrm{~N}_{3}$, and ${ }^{14} \mathrm{~N}_{2}{ }^{15} \mathrm{~N}_{2}$ isotopomers. The $\operatorname{CCSD}(\mathrm{T}) / \mathrm{cc}-\mathrm{pVQZ}$ harmonic and fundamental frequencies are presented in Table 2. CCSD(T)/cc-pCVQZ harmonic frequencies for each isotopomer were obtained with the CCSD(T)/cc-pCVQZ quadratic force field given in Table 1. Best Estimates for the gas-phase fundamental frequencies were then obtained by adding the $\mathrm{CCSD}(\mathrm{T}) / \mathrm{cc}-\mathrm{pVQZ}$ anharmonic correction to the CCSD(T)/cc-pCVQZ harmonic frequency. These frequency values are also given for each isotopomer in Table 2 . We note that the first two isotopomers have $T_{d}$ symmetry, the next two have $C_{3 v}$ symmetry, and the last one has $C_{2 v}$ symmetry. However, we have ordered the modes for each isotopomer so that they are consistent with the parent ${ }^{14} \mathrm{~N}_{4}$ isotopomer.

Considering the two $T_{d}$ isotopomers first, it is apparent that core-correlation increases the frequencies by between 3.4 to $6.3 \mathrm{~cm}^{-1}$ for ${ }^{14} \mathrm{~N}_{4}$ and by between 3.2 to 6.2 $\mathrm{cm}^{-1}$ for ${ }^{15} \mathrm{~N}_{4}$, which is somewhat less than what has been calculated for the $\mathrm{N}_{2}$ molecule [26]. This is to be expected, however, since the N-N bonds in $T_{d} \mathrm{~N}_{4}$ are single bonds whereas the N-N linkage in $\mathrm{N}_{2}$ is a triple bond. Further the difference in the type of N-N bonds is also evident by some of the anharmonic corrections. For the ${ }^{14} \mathrm{~N}_{4}$ isotopomer, anharmonic corrections for the two lowest energy modes are 16.9 and $18.4 \mathrm{~cm}-1$, which is significantly less than that for the $\mathrm{N}_{2}$ molecule, $28.6 \mathrm{~cm}-1$ [27]. However, the correction for the highest energy mode is $32.6 \mathrm{~cm}^{-1}$, which is probably a result of stretching (or compressing) all six bonds in $T_{d} \mathrm{~N}_{4}$ simultaneously. Nonetheless, the $\mathrm{t}_{2}$ mode is of most interest since it is the only one that is IR active [1].

Based on experience [17-20], we conservatively expect the Best Estimate fundamental frequencies to be accurate to within $\pm 4 \mathrm{~cm}^{-1}$, with the Best Estimate probably being somewhat too high relative to the gas-phase value since the main remaining error is probably neglect of higher-order electron correlation effects beyond 
what is included at the $\operatorname{CCSD}(\mathrm{T})$ level of theory. Thus, taken in this light, our prediction for the $t_{2}$ mode for ${ }^{14} \mathrm{~N}_{4}$ of $935.3 \mathrm{~cm}^{-1}$ would tend to support the experimental assignment, $936.7 \mathrm{~cm}^{-1}$, suggested in Ref. [13], keeping in mind that matrix effects can shift this somewhat as well. However, the residual error in our predicted isotopic shift

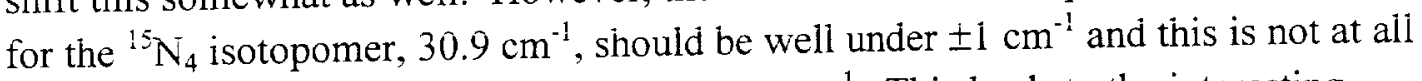
consistent with the experimental value of $36.7 \mathrm{~cm}^{-1}$. This leads to the interesting conclusion where we cannot definitively rule out an isotopomer of $T_{d} \mathrm{~N}_{4}$ for one of the bands observed in Ref. [13], but we can definitively say that both bands cannot be due to different isotopomers of $T_{d} \mathrm{~N}_{4}$. However, it seems unlikely that one of the bands is due to an isotopomer of $T_{d} \mathrm{~N}_{4}$ and the other band due to a different as yet unassigned molecule. More likely is that the two bands belong to ${ }^{14} \mathrm{~N}$ and ${ }^{15} \mathrm{~N}$ isotopomers of the same molecule, but this molecule is most definitely not $T_{d} \mathrm{~N}_{4}$.

Examination of the Best Estimate fundamental frequencies for the remaining isotopomers shows that of the bands originating from the $t_{2}$ mode in the ${ }^{14} \mathrm{~N}_{4}$ parent isotopomer, none of them are as low in frequency as the corresponding band in the ${ }^{15} \mathrm{~N}_{4}$ isotopomer. In other words, the largest isotopic shift occurs between the ${ }^{14} \mathrm{~N}_{4}$ and ${ }^{15} \mathrm{~N}_{4}$ isotopomers, so it is not possible that a mixed isotopomer accounts for the experimentally observed isotopic shift. From consideration of the data contained in Table 2 , there is little doubt that $T_{d} \mathrm{~N}_{4}$ has yet to be synthesized and observed.

For completeness, anharmonic constants according to the convention for spherical tops [28-30] are presented for ${ }^{14} \mathrm{~N}_{4}$ and ${ }^{15} \mathrm{~N}_{4}$ in Table 3. It is hoped that the data presented in Tables 2 and 3 will be useful in the ultimate synthesis and detection of the high energy density material $T_{d} \mathrm{~N}_{4}$.

\section{B. Binding Energy Relative to Two $\mathrm{N}_{2}$ Molecules}

In the decade since $T_{d} \mathrm{~N}_{4}$ was first identified as a high energy density material, techniques for computing highly accurate thermochemistry have evolved significantly. In many cases it is possible to compute these quantities more accurately than can be obtained from experiment, with the exception of high resolution spectroscopy where this approach is applicable. The more sophisticated approaches, for example, take into account extrapolation to the one-particle basis set limit, core-valence correlation, and Darwin mass-velocity and spin-orbit relativistic corrections. In the present study we have utilized the W2 approach, which has been shown to yield atomization energies and heats of formation to better than $\pm 0.5 \mathrm{kcal} / \mathrm{mol}$ for a set of 14 benchmark molecules [31]. The interested reader is referred to Ref. [31] for details of the W2 approach. The data used in the W2 approach are compiled in Table 4 for the atomization reactions of $\mathrm{N}_{2}$ and $T_{d} \mathrm{~N}_{4}$ and for the dissociation of $T_{d} \mathrm{~N}_{4}$ into two $\mathrm{N}_{2}$ molecules. CCSD(T)/cc-pVQZ reference geometries were used in these calculations.

On examination of the data in Table 4, the first point to note is the excellent agreement between experiment and W2 theory for the $\mathrm{N}_{2}$ molecule (being the reference compound, $\Delta \mathrm{H}_{\mathrm{f}, 298}$ is $0.0 \mathrm{kcal} / \mathrm{mol}$ ). This excellent agreement indicates that the $\mathrm{W} 2$ value for the heat of formation of $T_{d} \mathrm{~N}_{4}$ and for its dissociation into two $\mathrm{N}_{2}$ molecules should be accurate to better than $\pm 0.5 \mathrm{kcal} / \mathrm{mol}$. Thus the important result from Table 4 is that the 
dissociation of $T_{d} \mathrm{~N}_{4}$ into two $\mathrm{N}_{2}$ molecules is exothermic by $182.22 \pm 0.5 \mathrm{kcal} / \mathrm{mol}$ at $0 \mathrm{~K}$ or $180.64 \pm 0.5 \mathrm{kcal} / \mathrm{mol}$ at $298 \mathrm{~K}$. These values are in reasonable agreement with earlier calculations $[1,12]$, but their uncertainty is smaller. Nonetheless, the important point is that they confirm beyond doubt that $T_{d} \mathrm{~N}_{4}$ is indeed a high energy density molecule.

\section{Conclusions}

A highly accurate quartic force field has been computed for the high energy density material $T_{d} \mathrm{~N}_{4}$. The $\operatorname{CCSD}(\mathrm{T})$ electron correlation technique has been used in conjunction with the cc-pVQZ and cc-pCVQZ basis sets. The CCSD(T) quartic force field has been used to compute accurate anharmonic corrections to the fundamental vibrations of $T_{d} \mathrm{~N}_{4}$ including all ${ }^{14} \mathrm{~N}$ and ${ }^{15} \mathrm{~N}$ isotopomers. After correcting these for corevalence correlation, best estimate fundamental vibrational frequencies have been obtained. The isotopic shift between ${ }^{14} \mathrm{~N}_{4}$ and ${ }^{15} \mathrm{~N}_{4}$ for the one IR active mode shows beyond doubt that the bands observed in a matrix isolation experiment $[\mathrm{xx}]$ cannot be due to different isotopomers of $T_{d} \mathrm{~N}_{4}$. It is hoped, however, that the improved fundamental vibrational frequency predictions for all of the ${ }^{14} \mathrm{~N}$ and ${ }^{15} \mathrm{~N}$ isotopomers of $T_{d} \mathrm{~N}_{4}$ reported herein will be useful in the ultimate identification of $T_{d} \mathrm{~N}_{4}$.

W2 theory has also been applied to the thermochemistry of $T_{d} \mathrm{~N}_{4}$, allowing for the prediction of an improved dissociation energy with respect to two $\mathrm{N}_{2}$ molecules. Specifically, W2 theory yields dissociation energies of $182.22 \pm 0.5 \mathrm{kcal} / \mathrm{mol}$ and $180.64 \pm 0.5 \mathrm{kcal} / \mathrm{mol}$ at $0 \mathrm{~K}$ and $298 \mathrm{~K}$, respectively. These values show beyond doubt that $T_{d} \mathrm{~N}_{4}$ is indeed a high energy density molecule.

\section{Acknowledgments}

The authors gratefully acknowledge helpful discussions with Drs. Jeffrey Sheehy, and Chris Dateo. Funding for this research is gratefully acknowledged from the NASA Advanced Space Transportation Program. 


\section{References}

1. T. J. Lee and J. E. Rice, J. Chem. Phys. 94, 1215 (1991).

2. M. M. Francl and J. P. Chesick, J. Phys. Chem. 94, 526 (1990).

3. W. J. Lauderdale, J. F. Stanton, and R. J. Bartlett, J. Phys. Chem. 96, 1173 (1992).

4. D. R. Yarkony, J. Am. Chem. Soc. 114, 5406 (1992).

5. M.N. Glukhovtsev and P. von R. Schleyer, Int. J. Quantum Chem. 198, 547 (1992).

6. K. M. Dunn and K. Morokuma, J. Chem. Phys. 102, 4904 (1995).

7. A. A. Korkin, A. Balkova, R. J. Bartlett, R. J. Boyd, and P.v.R. Schleyer, J. Phys. Chem. 100, 5702 (1996).

8. M.N. Glukhovtsev and S. Laiter, J. Phys. Chem. 100, 1569 (1996).

9. M.N. Glukhovtsev, H. Jiao, and P. von R. Schleyer, Inorg. Chem. 35, 7124 (1996).

10. S. A. Perera and R. J. Bartlett, Chem. Phys. Lett. 314, 381 (1999).

11. M. Bittererova, T. Brinck, and H. Ostmark, J. Phys. Chem. A104, 11999 (2000).

12. M. Bittererova, T. Brinck, and H. Ostmark, Chem. Phys. Lett. 340, 597 (2001).

13. J. P. Zheng, J. Waluk, J. Spanget-Larsen, D. M. Blake, and J. G. Radziszewski,

Chem. Phys. Lett. 328, 227 (2000).

14. T. J. Lee and C. E. Dateo, Chem. Phys. Lett. 345, 295 (2001).

15. F. Cacace, G. de Petris, and A. Troiani, Science 295, 480 (2002).

16. K. Raghavachari, G. W. Trucks, J. A. Pople, and M. Head-Gordon, Chem. Phys. Lett. 157, 479 (1989).

17. T. J. Lee and G. E. Scuseria in Quantum Mechanical Electronic Structure Calculations with Chemical Accuracy, Ed. S. R. Langhoff (Kluwer, Dordrecht, 1995), pp. 47-108.

18. T. J. Lee, J. M. L. Martin, and P. R. Taylor, J. Chem. Phys. 102, 254 (1995).

19. J. M. L. Martin, T. J. Lee, P. R. Taylor, and J.-P. Francois, J. Chem. Phys. 103, 2589 (1995).

20. B. J. Persson, P. R. Taylor, and T. J. Lee, J. Chem. Phys. 107, 5051 (1997).

21. T. H. Dunning, J. Chem. Phys. 90, 1007 (1989).

22. D. E. Woon and T. H. Dunning, J. Chem. Phys. 103, 4572 (1995).

23. MOLPRO 96 is a package of ab initio programs written by H.-J. Werner and P.J.

Knowles, with contributions from J. Almll"of, R.D. Amos, M.J.O. Deegan, S.T. Elbert,

C. Hampel, W. Meyer, K. Peterson, R. Pitzer, A.J. Stone, and P.R. Taylor.

24. A. P. Rendell, T. J. Lee, and P. R. Taylor, J. Chem. Phys. 92, 7050 (1990).

25. SPECTRO, version 1.0 (1989), written by J. F. Gaw, A. Willetts,

W. H. Green, and N. C. Handy.

26. Core-valence for N2; Jan do you have the reference for this??

27. K. P. Huber and G. Herzberg, Constants of Diatomic Molecules (Van Nostrand

Reinhold, New York, 1979).

28. K. T. Hecht, J. Mol. Spectrosc. 5, 355 (1960).

29. J. Herranz, J. Mol. Spectrosc. 6, 343 (1961).

30. F. W. Birss, Mol. Phys. 31, 491 (1976).

31. J. M. L. Martin, W2 theory; Jan please input. 
Table 1. $\operatorname{CCSD}(\mathrm{T})$ equilibrium geometry and force fields for $T_{d} \mathrm{~N}_{4}{ }^{a}$

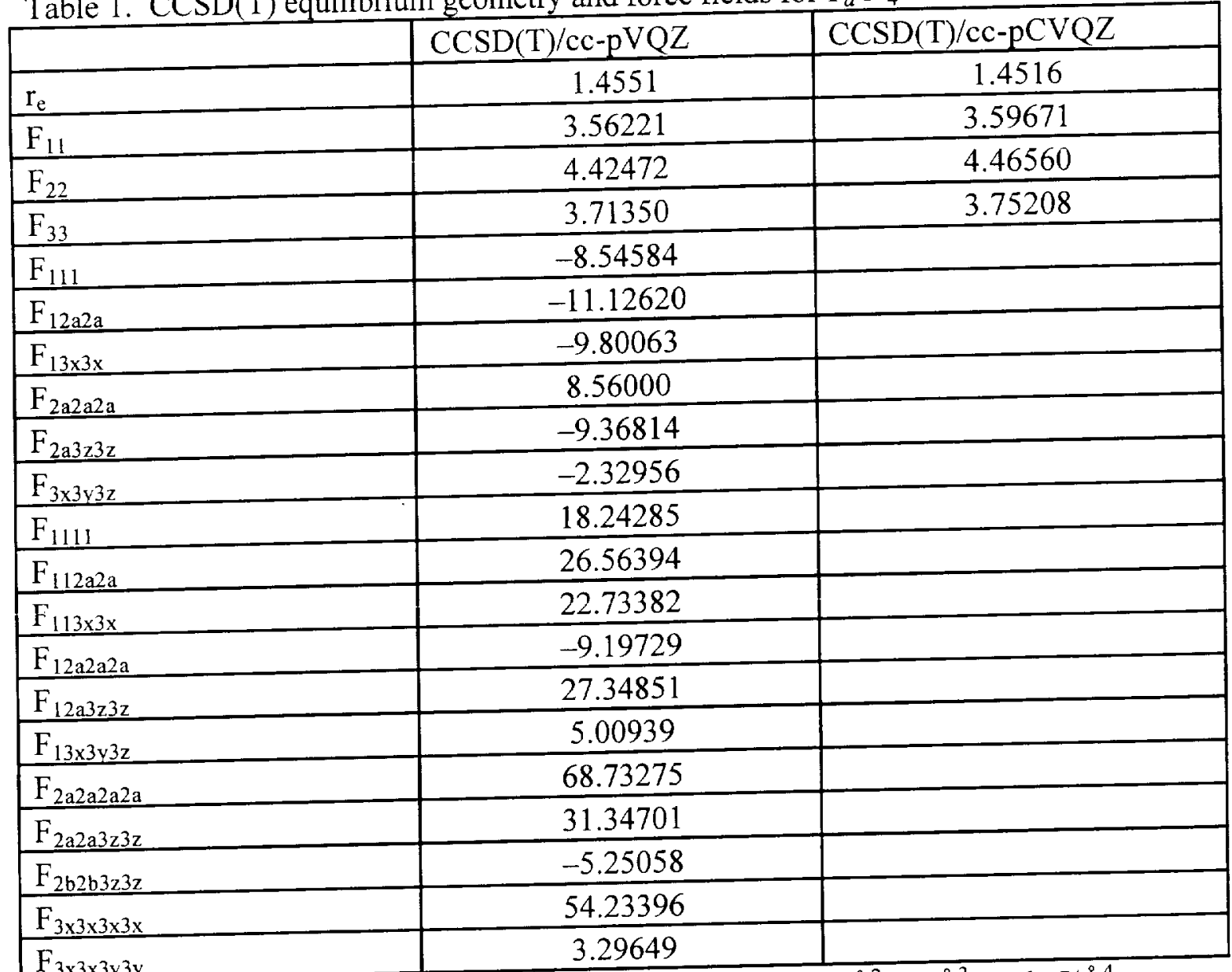

$F_{3 \times 3 \times 3 \times 3 v}$

${ }^{a}$ Units for quadratic, cubic, and quartic force constants are $\mathrm{aJ} / \AA^{2}, \mathrm{aJ} / \AA^{3}$, and aJ $/ \AA^{4}$, respectively. The equilibrium bond distance is in units of $\AA$. Only the unique symmetry internal coordinate force constants are given. See Ref. [xx] for symmetry relationships needed to complete the force field. 
Table 2. Theoretical harmonic, fundamental, and best estimate vibrational frequencies for the various isotopomers of $T_{d} \mathrm{~N}_{4}{ }^{\mathrm{a}}{ }^{\mathrm{a}}$

\begin{tabular}{|l|c|c|c|c|}
\hline Molecule/Mode & \multicolumn{2}{|c|}{$c c-p V Q Z$} & $c c-p C V Q Z$ & Best Estimate \\
\hline${ }^{14} \mathrm{~N}_{4}$ & $\omega$ & $v$ & $\omega$ & $v$ \\
\hline $\mathrm{a}_{1}$ & 1314.2 & 1281.6 & 1320.5 & 1287.9 \\
\hline $\mathrm{t}_{2}$ & 948.8 & 930.4 & 953.7 & 935.3 \\
\hline $\mathrm{e}$ & 732.3 & 715.4 & 735.7 & 718.8 \\
\hline${ }^{15} \mathrm{~N}_{4}$ & & & & \\
\hline $\mathrm{a}_{1}$ & 1269.7 & 1239.3 & 1275.9 & 1245.5 \\
\hline $\mathrm{t}_{2}$ & 916.7 & 899.6 & 921.5 & 904.4 \\
\hline $\mathrm{e}$ & 707.6 & 691.8 & 710.8 & 695.0 \\
\hline${ }^{14} \mathrm{~N}_{3}{ }^{15} \mathrm{~N}$ & & & & \\
\hline $\mathrm{a}_{1}$ & 1303.6 & 1271.5 & 1309.9 & 1277.8 \\
\hline $\mathrm{e}$ & 944.9 & 926.8 & 949.8 & 931.7 \\
\hline $\mathrm{a}_{1}$ & 932.3 & 914.4 & 937.2 & 919.3 \\
\hline $\mathrm{e}$ & 726.1 & 709.5 & 729.4 & 712.8 \\
\hline${ }^{14} \mathrm{~N}^{15} \mathrm{~N}_{3}$ & & & & \\
\hline $\mathrm{a}_{1}$ & 1281.4 & 1250.4 & 1287.6 & 1256.6 \\
\hline $\mathrm{a}_{1}$ & 932.3 & 914.7 & 937.1 & 919.5 \\
\hline $\mathrm{e}$ & 920.9 & 903.5 & 925.7 & 908.3 \\
\hline $\mathrm{e}$ & 713.7 & 697.6 & 717.0 & 700.9 \\
\hline${ }^{14} \mathrm{~N}_{2}{ }^{15} \mathrm{~N}_{2}$ & 932.4 & 914.6 & 937.2 & 919.4 \\
\hline $\mathrm{a}_{1}$ & 1292.7 & 1261.2 & 1298.9 & 1267.4 \\
\hline $\mathrm{b}_{1}$ & 940.9 & 922.9 & 945.7 & 912.0 \\
\hline $\mathrm{a}_{1}$ & 719.7 & 703.4 & 723.0 & 707.0 \\
\hline $\mathrm{b}_{2}$ & 907.2 & 929.6 & \\
\hline $\mathrm{a}_{2}$ & & 703.7 & 723.4 & \\
\hline $\mathrm{a}_{1}$ & & & & \\
\hline $\mathrm{a}_{1}$ & & & & \\
\hline
\end{tabular}

${ }^{a}$ Obtained at the CCSD(T) level of theory using the basis set indicated. All quantities are in units of $\mathrm{cm}^{-1}$. The ordering of the modes is designed to be consistent with the parent isotopomer. 
Table 3. Anharmonic constants for ${ }^{14} \mathrm{~N}_{4}$ and ${ }^{15} \mathrm{~N}_{4} \cdot{ }^{\text {a }}$

\begin{tabular}{|l|c|c|}
\hline & ${ }^{14} \mathrm{~N}_{4}$ & ${ }^{15} \mathrm{~N}_{4}$ \\
\hline $\mathrm{x}_{11}$ & -2.691 & -2.512 \\
\hline $\mathrm{x}_{21}$ & -12.162 & -11.354 \\
\hline $\mathrm{x}_{22}$ & 0.929 & 0.867 \\
\hline $\mathrm{x}_{31}$ & -10.009 & -9.344 \\
\hline $\mathrm{x}_{32}$ & -5.359 & -5.003 \\
\hline $\mathrm{x}_{33}$ & -1.589 & -1.483 \\
\hline $\mathrm{g}_{22}$ & -5.578 & -5.207 \\
\hline $\mathrm{g}_{33}$ & -0.830 & -0.775 \\
\hline $\mathrm{t}_{23}$ & 0.400 & 0.373 \\
\hline $\mathrm{t}_{33}$ & 0.063 & 0.059 \\
\hline
\end{tabular}

${ }^{a}$ All constants are in units of $\mathrm{cm}-1$. Obtained at the CCSD(T)/cc-pVQZ level of theory. 
Table 4. Thermochemical data $(\mathrm{kcal} / \mathrm{mol})$ for the noted reactions used in the $\mathrm{W} 2$ level of

\begin{tabular}{|c|c|c|c|}
\hline & $2 \mathrm{~N} \rightarrow \mathrm{N}_{2}$ & $4 \mathrm{~N} \rightarrow \mathrm{N}_{4}$ & $2 \mathrm{~N}_{2} \rightarrow \mathrm{N}_{4}$ \\
\hline $\begin{array}{l}\text { SCFlimit- } \\
\text { SCF/AV } 5 Z\end{array}$ & 0.04 & 0.01 & -0.07 \\
\hline $\begin{array}{l}\text { CCSDlimit- } \\
\text { CCSD/AV } 5 Z\end{array}$ & 1.64 & 3.39 & 0.12 \\
\hline (T)limit-(T)/VQZ & 0.26 & 0.77 & 0.24 \\
\hline SCF & 119.70 & 8.50 & -230.91 \\
\hline CCSD & 98.23 & 238.41 & 41.95 \\
\hline$(\mathrm{T})$ & 9.45 & 28.02 & 9.11 \\
\hline CORE & 0.73 & 0.31 & -1.14 \\
\hline DMV & -0.11 & -0.19 & 0.04 \\
\hline $\mathrm{S}-\mathrm{O}$ & 0.00 & 0.00 & 0.00 \\
\hline Equilibrium Total & 228.00 & 275.05 & -180.95 \\
\hline ZPVE & 3.36 & 7.99 & 1.27 \\
\hline ZP Total & 224.64 & 267.06 & -182.22 \\
\hline$\Delta \mathrm{H}_{0}$ & 0.42 & 183.06 & 182.22 \\
\hline$\Delta \mathrm{H}_{298}$ & 0.42 & 181.48 & 180.64 \\
\hline
\end{tabular}

${ }^{\mathrm{a}}$ See Ref. [xx] for a description of the W2 approach to thermochemical calculations. 